\title{
Desgaste profesional, estrés y de la profesión en enfermería
}

M. Rosa Girbau Garciaa, Jordi Galimany Masclans ${ }^{\mathrm{b}}$ y Eva Garrido Aguilarc

a Enfermera y Doctora en Ciencias Enfermeras, Profesora Titular de la Escuela Universitaria de Enfermería de la Universidad de Barcelona, Barcelona, España

b Enfermero y Master en Liderazgo y Gestión de Servicios, Profesor Colaborador Permanente de la Escuela Universitaria de Enfermería de la Universidad de Barcelona, Barcelona, España

c Profesora Colaboradora de la Escuela Universitaria de Enfermería de la Universidad de Barcelona, Barcelona, España

\section{Los sistemas sanitarios y sus profesionales se justifican en la medida} que están al servicio de las personas y son capaces de dar respuesta a las necesidades de salud y a las expectativas de la comunidad'.

\section{El reconocimiento a las enfermeras como un valor humano}

y profesional de primera magnitud dentro del sistema de salud de cualquier país, y en general a las profesiones sanitarias, se caracteriza por el compromiso, la vocación y el espíritu de servicio, y el mayor activo del sistema son los recursos humanos.

\section{Introducción}

El informe sobre los Recursos Humanos Sanitarios en España y la Unión Europea, presentado en el año 2007 por el Consejo General de Enfermería de España², comparó los recursos de enfermería existentes en España con los del resto de países de nuestro entorno cercano. La ratio en la Europa de los 27 era de 808 enfermeras por cada 100.000 habitantes, dato muy superior a la ratio española, que era de 532 enfermeras.

Estos datos dan una idea muy clara del esfuerzo personal y profesional que realizan estas profesionales para la consecución de sus objetivos asistenciales, dando un valor añadido a su profesión, ya que en todo sistema de salud esos recursos humanos son un elemento esencial para dispensar los cuidados a la población sana y/o enferma.
Además, la precariedad laboral de las enfermeras es alta y la situación de inserción laboral de los nuevos titulados es aún muy deficiente, lo que añade más elementos de estrés y desgaste profesional.

\section{Desgaste profesional}

El síndrome de desgaste profesional, conocido también como "burnout" o "síndrome del quemado", fue descrito en 1974 por Freudenberger como "un estado de fatiga o frustración que se produce por la dedicación a una causa, forma de vida o relación que no produce el esperado refuerzo". En 1976 Maslach lo definió como "una respuesta inadecuada a un estrés laboral crónico, cuyos rasgos principales son el agotamiento físico y/o psicológico". Este síndrome parece afectar en mayor medida a las profesiones de ayuda, en las cuales se gestionan emociones intensas de enfermedad, dolor y/o sufrimiento, sobre todo a los profesionales de la salud ${ }^{3}$.

En el ámbito laboral, el burnout aparece por el estrés laboral continuo y por la ausencia de expectativas de superación del mismo. Se caracteriza por tres componentes: agotamiento emocional, despersonalización y falta de realización personal ${ }^{4}$, con manifestaciones tanto físicas como psíquicas, y genera insatisfacción, absentismo y disminución de la calidad del trabajo, lo que afecta tanto al trabajador como a la familia, al usuario y a la institución ${ }^{5}$.

Cuando una enfermera manifiesta que está "quemada", refleja que la situación laboral, familiar o social la ha sobrepasado, agotando su capacidad de reacción y respuesta, y es el precio que se paga por intentar satisfacer el rol profesional y el personal ${ }^{6}$. Hasta llegar al agotamiento emocional, la persona pasa por diferentes fases, desde la actitud de entusiasmo y altas expectativas, a la fase de estancamiento y frustración, pasando por la apatía o indiferencia hacia el trabajo, lo que repercute negativamente en la calidad asistencial ${ }^{7}$.

Diversos estudios ${ }^{8-9}$ realizados en personal de enfermería de atención primaria de salud (APS) nos confirman la percepción de que el estrés está fuertemente asociado con el burnout y con efectos adversos para la salud 
y la satisfacción de las enfermeras. En un amplio análisis realizado por Blegen $^{10}$ para describir la relación entre la satisfacción laboral de las enfermeras americanas y las variables que más influían en ésta, los resultados revelaron que el estrés era la variable que tenía una mayor correlación con la satisfacción, seguida del compromiso con la organización; en el estudio destacaba la complejidad de identificar la multiplicidad de variables que pueden influir en el estrés.

Otros estudios realizados en Europa ${ }^{11-12}$ constataron un alto grado de agotamiento emocional y despersonalización con una correlación importante con el conflicto de rol y la salud psicosomática de las enfermeras, y la influencia que el estrés puede tener en el rendimiento laboral, en la eficiencia organizacional y en los cuidados ofrecidos a los pacientes, con un coste añadido para el sistema de salud.

Por otra parte, un estudio ${ }^{13}$ realizado para conocer la prevalencia de burnout en las enfermeras en España concluyó que el $28,8 \%$ de estas profesionales tenían un alto grado de agotamiento emocional, el 32,2\% un alto grado de despersonalización y el 92,8\% tenían un grado moderado de falta de realización personal.

Según otro estudio ${ }^{14}$ realizado en personal sanitario hispanoamericano y español para conocer la influencia de factores personales, profesionales

y transnacionales, la prevalencia de burnout fue mayor en España y en Argentina que en el resto de países estudiados. Los autores del estudio argumentaron que las diferencias transnacionales apoyan la influencia del contexto social en la génesis de este síndrome, y añaden que las condiciones laborales y personales que podrían considerarse más "duras" pueden ser percibidas como menos estresantes en los países con niveles

\section{El síndrome de desgaste profesional, conocido también como} "burnout" o "síndrome del quemado", fue descrito en 1974 por Freudenberger como "un estado de fatiga o frustración que se produce por la dedicación a una causa, forma de vida o relación que no produce el esperado refuerzo"

de bienestar inferiores, dando mayor importancia al contexto macrosocial y familiar que al laboral.

\section{Estrés laboral}

El estrés laboral se caracteriza por altos niveles de excitación y angustia, con la sensación de no poder enfrentarse a la situación, y puede afectar al ámbito de la productividad con una disminución de la satisfacción y un aumento del absentismo laboral ${ }^{15-17}$

Las experiencias que pueden provocar situaciones de estrés en la profesión enfermera son diversas: la brevedad en la duración de los contratos, la falta de un horario fijo, los turnos con semanas de 60 h y otras de 20 h, la precariedad en el trabajo, la rotación de centros asistenciales, la falta de expectativas de promoción, la dificultad de obtener permisos de maternidad y paternidad por la inestabilidad contractual, la sobrecarga de trabajo, los recursos inadecuados y los factores ambientales, junto con las características personales ${ }^{18-20}$. 
de la capacidad y la autonomía en la planificación de la vida personal y familiar. También ha aumentado de forma significativa el número de centros y servicios donde han trabajado, lo que demuestra un alto índice de rotación laboral.

\section{El estrés laboral se caracteriza por altos niveles de excitación y angustia, con la sensación de no poder enfrentarse a la situación, y puede afectar al ámbito de la productividad con una disminución de la satisfacción y un aumento del absentismo laboral}

En un informe realizado en el año 2008, el Consell Català de la Professió Enfermera ${ }^{22}$ constató que la precariedad laboral influía negativamente en las dinámicas de los equipos, y añadía, además, que tenía un impacto poco favorable en los resultados clínicos de los pacientes y en la continuidad de los procesos asistenciales. Al mismo tiempo, el informe concluía que la enfermera se sentía menos satisfecha con su actividad profesional, disminuyendo su calidad de vida ${ }^{23}$

Esta realidad demuestra que a pesar de haberse incrementado el número de los contratos fijos en el personal de enfermería, éstos siguen siendo bajos, lo que refleja que la situación contractual de las enfermeras obedece más a criterios desarrollo de una práctica asistencial, de colaboración entre los distintos profesionales y el trabajo en equipo, para ofrecer a los ciudadanos un servicio de calidad basado en la continuidad de los cuidados y la eficiencia del sistema ${ }^{25}$. La intermitencia y la falta de estabilidad contractual pueden ocasionar la pérdida de referente de la enfermera y no facilitan el seguimiento adecuado del paciente con una optimización del tiempo y los recursos.

Abandono de la profesión
y déficit de enfermeras
Las estadísticas revelan que las
enfermeras de APS en Cataluña ${ }^{26}$ tienen
una media de edad de 47 años, cifra
similar a la del resto de países europeos.
Este aspecto es de gran interés en el
análisis del abandono y la escasez de
profesionales en los últimos años.
Esta realidad sociodemográfica de
las enfermeras en nuestro país coincide
con los datos presentados en diferentes

APS, pero sí hay una clara coincidencia en todos ellos, y es que la satisfacción es un elemento clave ${ }^{28-29}$, considerando que las enfermeras que están insatisfechas tienen un $65 \%$ más de probabilidad de abandonar el trabajo que las que están satisfechas.

Según Ballester ${ }^{30}$, el déficit de enfermeras en los últimos años en Cataluña no se produce de la misma forma que en el resto de comunidades autónomas, y representa un 27\% menos que la media europea. Este autor profundiza en el análisis de los motivos de este déficit de profesionales, y añade a su vez que esta situación se verá agravada por el paso actual de la titulación de enfermería de 3 a 4 años, con lo que durante un año no se titulará ninguna enfermera; es decir, una promoción: 1.800 enfermeras en Cataluña. No obstante, cabe destacar que durante ese periodo de transición la finalización de los estudios de la diplomatura de enfermería se producirá de forma gradual, ya que habrá alumnos repetidores. De acuerdo con Esteve ${ }^{31}$, en los próximos años faltarían en Cataluña 15.000 enfermeras, el 35\% de las existentes en la actualidad.

Asimismo aparecen coincidencias en los diferentes estudios realizados a nivel internacional ${ }^{32-40}$-Australia, Canadá, Estados Unidos, Jordania, Irán- en relación con los factores que pueden influir en la satisfacción, considerando la flexibilidad en el horario de trabajo, la falta de políticas organizativas y el reconocimiento profesional como la clave en la decisión de abandonar el trabajo como consecuencia de la falta de satisfacción.

Al mismo tiempo, en un estudio de casos de abandono de la profesión hecho sobre unas 40.000 enfermeras procedentes de once países europeos se puso de manifiesto la relación evidente entre la satisfacción en el trabajo y la intención de abandonar la profesión; cuanto menor era aquélla, mayor era la intención de abandonarla. Concluyeron que los motivos principales estaban relacionados con unas condiciones laborales deficientes, y que abandonan el trabajo para encontrar otro que les ofrezca un mejor horario laboral, mayor remuneración, más seguridad que en el entorno del sector de salud, o que les permita cuidar de su casa y de su familia ${ }^{41-42}$. estudios a nivel internacional, y es especialmente importante el abandono de la profesión entre los grupos de mayor edad ${ }^{27-28}$. En el análisis realizado por diferentes investigadores sobre estudios recientes se concluye que hay importantes lagunas en la literatura, en relación con los factores que influyen en la retención de las enfermeras en la gestores de nuestro sistema de salud, que va dirigido a potenciar el 


\section{Conclusiones}

Los estudios revisados ponen de relieve la relación que existe entre el desgaste profesional, el estrés y el abandono de la profesión de las enfermeras asistenciales. La imagen enfermera como profesión femenina ha marcado el rol de cuidadora de la unidad familiar. También es cierto que las enfermeras abandonan temporalmente la profesión por razones de maternidad, y en muchas ocasiones su incorporación al trabajo supone un problema añadido a su responsabilidad como madres, con lo que existe la posibilidad de que aparezcan problemas de salud asociados al estrés laboral y al desgaste profesional.

No se debe olvidar que ese potencial profesional que tienen las enfermeras debe ser gestionado con objetividad y sensibilidad, atendiendo a sus particularidades laborales y personales. Por ello parece necesario facilitar y apoyar estrategias que permitan un desarrollo armonizado de su carrera profesional, con la asunción del rol familiar, con el interés de ofrecer un mejor cuidado del paciente y de su familia, ya que la satisfacción de las enfermeras es clave en los sistemas de salud, destacando la naturaleza de los cuidados que éstas proporcionan al individuo, a la familia y a la comunidad para promover, prevenir, mejorar y restaurar la salud a lo largo del ciclo vital.

\section{Bibliografía}

1. Pasarin MI, Millar R, Segura A. Aportaciones de la atención primaria y la salud pública al desarrollo de la salud comunitaria. Aten Primaria. 2008;40(3):115-7.

2. Consejo General de Colegios Oficiales de Enfermería de España. Informe sobre Recursos Humanos sanitarios en España y la Unión Europea. Madrid: Consejo General de Enfermería; 2007.

3. Lomeña JA, Campaña FM, Nuevo G, Rosas D, Berrocal A, García F. Burnout y satisfacción laboral en Atención Primaria. Med Familia. 2004;5(3):147-55.

4. Villagrán SA, Cañas I, Rodríguez Y, Lagares $\mathrm{R}$, Lahoz B, Barros C. Técnicas de grupo nominal para conocer alternativas que prevengan o mejoren el desgaste profesional en los trabajadores de un Centro de Salud. Med Familia. 2005;6(2):24-31.

5. Caballero MA, Bermejo F, Nieto R, Caballero F. Prevalencia y factores asociados al burnout en un área de salud. Aten Primaria. 2001;27(5):313-17.

6. De Pablo CL. Síndrome de burnout en los profesionales sanitarios. Nursing. 2007;25(8);60-5.

7. Carmona FJ. Clima social laboral y síndrome de Burnout en profesionales de enfermería. Enferm Científica. 2002;244-45:82-87.
8. Martín MC. Estrés relacionado con el trabajo (modelo de demanda-control-apoyo social) y alteraciones en la salud: una revisión de la evidencia existente. Enferm Intensiva. 2007;18(4):168-81.

9. Fernández JA, Fernández M, Martín R, Rödel A. Estrés laboral y calidad de vida en sanitarios de atención primaria: una prueba de la validez del cuestionario PECVEC. Aten Primaria. 2007;39(8):425-31.

10. Blegen MA. Nurses' Job Satisfaction: A Meta-analysis of related variables. Nurs Research. 1993;42(1):36-42

11. Piko BF. Burnout, role conflict, job satisfaction and psychosocial health among Hungarian health care staff: A questionnaire survey. Int J Nurs Stud. 2006:43(3):311-18.

12. Hawksley B. Work-related stress, work/life balance and personal life coaching. Br J Community Nurs. 2007;12(1):34-6.

13. Soto R, Santamaría MI. Prevalencia del burnout en la enfermería de atención primaria. Enf Clin. 2005; 15(3):123-30

14. Grau A, Flichtentrei D, Suñer R, Prats M, Braga F. Influencia de factores personales, profesionales y transnacionales en el síndrome de burnout en personal sanitario hispanoamericano y español (2007). Rev Esp Salud Pública. 2009;83:215-30

15. Escribá V, Más R, Cárdenas M, Pérez S Validación de la escala de estresores laborales en personal de enfermería: "The Nursing Stress Scale". Gac Sanit. 1999;13(3):191-200.

16. Brosschot JF, Pieper S, Thayer JF. Expanding stress theory: Prolonged activation and perseverative cognition. Psychoneuroendocrinology. 2005;30:1043-49

17. Casas J, Repullo JR, Lorenzo S. Estrés laboral en el medio sanitario y estrategias adaptativas de afrontamiento. Rev Cal Asistenc. 2002;17(4):237-46.

18. Moncada S. Estrès, factors psicosocials i salut. Institut Sindical de Treball, Ambient i Salut. Jornada Riscos psicosocials. Barcelona; 2003.

19. Pades A, Homar C. Estrés laboral y burnout en Enfermería. Metas de Enferm. 2006;9(3):67-72.

20. Sveinsdóttir H, Biering P, Ramel A.

Occupational stress, job satisfaction, and working environment among Icelandic nurses: a crosssectional questionnaire survey. Int J Nurs Stud. 2006;43(7):875-89.

21. Casanovas I, Escuredo B, Esteve J. Trayectoria laboral de las enfermeras de una escuela durante los 4 años siguientes a la graduación: 2000-2004. Enferm Clin. 2006;16(5):238-43

22. Consell Català de la Professió Infermera. Informe executiu: "Limpacte de la precarietat laboral del personal d'Infermeria en els resultats clínics i de productivitat". Comissió de l'exercici. Grup Estabilitat/Precarietat. Barcelona; 2008.

23. Lizán L. La calidad de vida relacionada con la salud. Aten Primaria. 2009;41(7):411-6.

24. San Martín L, D’Amour D, Ferrada M. La colaboración entre los profesionales de la salud (I) Contexto, Dinámica y Elementos Determinantes. Rev Rol Enf. 2006;29(6):425-30.

25. Peya M. Prestar servicios a la comunidad: Las enfermeras liderando la atención primaria de salud. Nursing. 2008;26(4):6.

26. Generalitat de Catalunya. Departament de Salut. Planificació i Avaluació. Mapa sanitari, sociosanitari i de salut pública. Barcelona; 2008.

27. Storey C, Cheater F, Ford J, Leese B. Retaining older nurses in primary care and the community. J Adv Nurs. 2009;65(7):1400-11.
28. Tourangeau AE, Cranley LA. Nurse intention to remain employed: understanding and strengthening determinants. J Adv Nur. 2006;55(4):497-509.

29. Storey C, Cheater F, Ford J, Leese B. Retention of nurses in the primary and community care workforce after the age of 50 years: Database analysis and literature review. J Adv Nurs. 2009;65(8):1596-605

30. Ballester D. La escasez de enfermeras en los próximos 15 años en Cataluña. 2009. [acceso 6 Novi 2010]. Disponible en: http://www.educaweb. com/noticia/2009/04/20/escasez

31. Esteve J. Demanda Universitària i manca d'Infermeres a Catalunya. Consell de Col.legis de Diplomats en Infermeria de Catalunya. Barcelona: 2008.

32. Cowwin L. The effects of Nurses' Job satisfactions on retention: An Australian perspective J Nurs Adm. 2002;32(5):283-91.

33. Irvine DM, Evans MG. Job satisfaction and turnover among nurses: Integrating research findings across studies. Nurs Res. 1995;44(4):246-53

34. Tett RP, Meyer JP. Job satisfaction, organizational commitment, turnover intention, and turnover: Path analyses based on metaanalytic findings. Personnel Psychology. 2006;46(2):259-93

35. Brush BL, Vasupuram R. Nurses, nannies and caring work: importation, visibility and marketability. Nursing Inquiry. 2006;13(3):181-85. 36. Castle NG, Engberg J, Anderson RA. Job satisfaction of nursing home administrators and turnover. Medical Care Research and Review. 2007;64(2):191-211.

37. Molinari DL, Monserud MA. Rural nurse job satisfaction. Rural and Remote Health 2008; 8: 1055. [acceso 6 Novi 2010]. Disponible en: http:// www.rrh.org.au

38. Taunton RL, Bott MJ, Koehn ML, Miller P, Rindner E, Pace K, et al. The National database of nursing quality indicators. NDNQI-adapted index of work satisfaction. J Nurs Meas. 2004;12(2):101-22. 39. Hussami MAL. A study of nurses' job satisfaction: the relationship to organizational commitment, perceived organizational support, transactional leadership, transformational leadership, and level of education. European J Scient Research. 2008;22(2):286-95.

40. Khani A, Jaafarpour M, Dyrekvandmogadam A. Quality of nursing work life. J Clinic Diagn Research. 2008;2:1169-74.

41. Consejo Internacional de Enfermería. Contratación y retención de las enfermeras: formación de unos recursos humanos motivados. La iniciativa del análisis mundial de la enfermería. Doc temático 4. Suiza 2006. [acceso 6 Novi 2010]. Disponible en: http://www.icn.ch/global/ Issue4RetentionSP.pdf

42. Hasselhorn HM, Tackenberg P, Hans B. Working conditions and intent to leave the profession among nursing staff in Europe. NEXT. Nurses' early exit study. Report 7:2003. The National Institute for working life. Estocolmo, Suecia [acceso 10 Juli 2009]. Disponible en: http://www. next-study.net 